\title{
MicroRNA-361-3p regulates retinoblastoma cell proliferation and stemness by targeting hedgehog signaling
}

\author{
DAN ZHAO and ZHE CUI
}

\begin{abstract}
Department of Ophthalmology, The Third Affiliated Hospital of Qiqihar Medical University, Qiqihar, Heilongjiang 161000, P.R. China
\end{abstract}

Received May 07, 2018; Accepted November 07, 2018

DOI: $10.3892 /$ etm.2018.7062

\begin{abstract}
Retinoblastoma (RB) is the most common type of intraocular malignancy in children. During RB oncogenesis, sonic hedgehog $(\mathrm{SHH})$ is commonly differentially expressed. Additionally, microRNAs (miRs) are known to serve crucial roles as oncogenes or tumor suppressors. Specifically, miR-361-3p has been revealed to serve a vital role in cutaneous squamous cell carcinoma, cervical cancer, prostate cancer, colorectal cancer, gastric cancer, hepatocellular carcinoma, breast cancer and lung cancer. However, the role of miR-361-3p in RB and the potential molecular mechanisms involved remain unknown. Therefore, the current study aimed to determine the involvement of miR-361-3p in the development of RB by targeting SHH signaling. In the present study, miR-361-3p expression levels in RB tissue and serum samples obtained from 10 patients with RB, normal retinal tissue and serum samples obtained from 10 healthy controls, and two human RB cell lines (Y79 and Weri-Rb-1) were determined using reverse transcription-quantitative polymerase chain reaction. In addition, a cell counting kit- 8 assay, a cell transfection assay, a MTT assay, western blotting, a tumor sphere formation assay and a luciferase assay were used to assess the expression, function and molecular mechanism of miR-361-3p in human RB. It was demonstrated that miR-361-3p was significantly downregulated in RB tissues, RB serum and RB cell lines compared with normal retinal tissues and normal serum. The ectopic expression of miR-361-3p decreased RB cell proliferation and stemness. Furthermore, GLI1 and GLI3 were verified as potential direct targets of miR-361-3p. miR-361-3p was also revealed to exhibit a negative correlation with GLI1/3 expression in RB samples. Taken together, the results
\end{abstract}

Correspondence to: Dr Zhe Cui, Department of Ophthalmology, The Third Affiliated Hospital of Qiqihar Medical University, 27 Taishun Street, Qiqihar, Heilongjiang 161000, P.R. China E-mail: saja665008@126.com

Key words: microRNA-361-3p, retinoblastoma, proliferation, metastasis, glioma-associated oncogene homologue transcription factor 1, glioma-associated oncogene homologue transcription factor 3 , hedgehog signaling indicate that miR-361-3p functions as a tumor suppressor in the carcinogenesis and progression of RB by targeting $\mathrm{SHH}$ signaling. Thus, miR-361-3p should be further assessed as a potential therapeutic target for RB treatment.

\section{Introduction}

Retinoblastoma ( $\mathrm{RB}$ ) is the most prevalent type of primary malignant intraocular cancer of infancy and childhood, particularly in children $<5$ years old (1). It represents $\sim 4 \%$ of all pediatric malignancies (2). It is estimated that 5,000 cases of RB are diagnosed each year, worldwide, with 250-300 cases in the United States alone (2). The clinical manifestations of RB vary greatly with disease stage (3). In its earliest clinical stage, RB presents as a small transparent or slightly translucent lesion in the sensory retina (2). Larger tumors exhibit retinal blood vessel dilation or foci of chalk-like calcification (3). Often, RB of any size presents as leukocoria, also known as the white pupillary reflex, at more advanced stages (2). The survival rate of $\mathrm{RB}$ is $>95 \%$ in the United States, but is $50 \%$ worldwide as RB is often detected when it has invaded the orbit or brain (2). Current disease management includes chemotherapy, enucleation, laser therapy or cryotherapy (4). However, the most common treatment protocol includes chemotherapy combined with immunotherapy, which is cytotoxic to RB cells (5). However, this treatment produces various adverse effects, including the acceleration of RB progression, meaning that the development of other therapeutic methods, particularly those that target specific signaling pathways, is required $(4,6)$.

MicroRNAs (miRs) are small (20-25 nucleotides), noncoding RNAs that regulate the expression of certain target genes in various physiological and pathological processes (7). They serve key roles as oncogenes or tumor suppressors and thus regulate tumor progression and metastasis $(8,9)$. Several critical miRs have been identified in RB progression. Hsa-miR-373 may be involved in RB invasion and metastasis, while hsa-miR-125b and hsa-let-7b may function as tumor suppressors by co-regulating cell division protein kinase 6 , cell division cycle $25 \mathrm{~A}$ and lin-28 homolog A $(10,11)$. Furthermore. hsa-miR-181a may serve a key role in the cyclin-dependent kinase inhibitor 1B-regulated cell cycle pathway (10). Certain miRs, including hsa-miR-25, hsa-miR-18a and hsa-miR-20a may affect the progression of RB by coregulating BCL2-like 
1 (10). Furthermore, miR-497 functions as a tumor suppressor in RB carcinogenesis and progression (12). He et al (13), demonstrated the suppressive effect of miR-143 in RB by targeting CDK6. In addition, miR-31 and miR-200a expression was revealed to be significantly reduced in human $\mathrm{RBs}$ and was determined to mediate the regulation of $\mathrm{RB}$ proliferation (4). miR-125b is also significantly upregulated in $\mathrm{RB}$, promoting tumor growth and suppressing apoptosis (11). Castro-Magdonel et al (14), has recently revealed a miR landscape that exhibits a core-cluster of $30 \mathrm{miRs}$ in RB. miR-3613 was also identified as being a potential and critical downregulatory marker in RB (14). However, the regulatory mechanisms involving $\mathrm{miR}$ in $\mathrm{RB}$ are yet to be fully elucidated.

Accumulating evidence has indicated that miR-361 serves important roles in various types of human tumor, including cutaneous squamous cell carcinoma (15), cervical cancer (16), prostate cancer (17), colorectal cancer (17), gastric cancer (18), hepatocellular carcinoma (19), breast cancer (20) and lung cancer (21). Additionally, the positive expression of miR-361-5p indicates a better prognosis for patients with breast cancer (20). Furthermore, Chen et al (21) revealed that miR-361-3p suppresses tumor cell proliferation and metastasis by directly targeting Src homology 2 B adapter protein 1 in non-small cell lung cancer (NSCLC).

According to Chen et al (21), sonic hedgehog ( $\mathrm{SHH})$ signaling proteins are frequently expressed in RB and are associated with aggressive clinicopathological features, including optic nerve invasion, choroidal invasion, extraocular invasion and metastasis. The activation of SHH signaling is regulated by the glioma-associated oncogene homologue (GLI) transcription factors (GLI1, 2 and 3), which induce several downstream pathways, including ATP-binding cassette G2 (22). The SHH pathway has been demonstrated to be dysregulated in various different types of cancer, including basal cell carcinoma and medulloblastoma $(23,24)$. However, few studies have assessed the role of SHH signaling in RB (25).

As miRs are involved in neoplastic progression, the current study hypothesized that the SHH pathway may serve a key role in the development or progression of $\mathrm{RB}$ by regulating $\mathrm{miR}$ expression as it has been reported that miRs are involved in many types of invasive tumor by targeting SHH proteins (25). To the best of our knowledge, this is the first study to determine the involvement of miR-361-3p in RB by targeting $\mathrm{SHH}$ signaling.

\section{Materials and methods}

Clinical samples. The present study was performed under the approval of the Clinical Research Ethics Committee of The Third Affiliated Hospital of Qiqihar Medical University (Qiqihar, China), and written informed consent was obtained from each patient prior to surgical excision. RB tissues, RB serums and matched adjacent, non-tumorous normal tissues were collected from 10 patients with RB who did not receive any preoperative therapies, including radiotherapy and chemotherapy. All patients (aged from 1 to 30 months) underwent ophthalmectomy at The Third Affiliated Hospital of Qiqihar Medical University between 2014 and 2017. The clinical characteristics of the patients with RB included in the current study are presented in Table I. Non-tumorous normal tissues were obtained separately from an opposite quadrant of the tumor site ( $>5 \mathrm{~mm}$ from the margin of the tumor) to avoid RB contamination. Control serums were obtained for 10 healthy participants. Following surgery, fresh RB and serum specimens were immediately snap-frozen in liquid nitrogen and stored at $-80^{\circ} \mathrm{C}$ for further examination.

Reverse transcription-quantitative polymerase chain reaction $(R T-q P C R)$. Total RNA was extracted from RB cells or tissues using the TRIzol reagent (Invitrogen; Thermo Fisher Scientific, Inc., Waltham, MA, USA) according to the manufacturer's protocol. The experiment was performed as previously described (26). The Taqman miR Reverse Transcription kit (Applied Biosystems; Thermo Fisher Scientific, Inc.) was used. RT-qPCR analysis of miR was performed using the TaqMan miR assay system (Applied Biosystems, Foster City, CA, USA). Small nuclear RNA U6 was utilized as an endogenous control. The relative expression of mRNA was determined using a SYBR Green RT-qPCR assay (Invitrogen; Thermo Fisher Scientific, Inc.), with $\beta$-actin as an endogenous control. All RT-qPCR procedures were performed using an ABI 7500 thermocycler (Invitrogen; Thermo Fisher Scientific, Inc.). Relative expression was calculated using the $2^{-\Delta \Delta c q}$ method (27). The thermocycling conditions were as follows: $95^{\circ} \mathrm{C}$ for $2 \mathrm{~min}$, followed by 30 cycles of amplification at $94^{\circ} \mathrm{C}$ for $40 \mathrm{sec}$, annealing at $55^{\circ} \mathrm{C}$ for $40 \mathrm{sec}$ and extension at $72^{\circ} \mathrm{C}$ for $1 \mathrm{~min}$, with a final elongation step at $72^{\circ} \mathrm{C}$ for $10 \mathrm{~min}$. The primers utilized were as follows: GLI1 forward, 5'-TTC CTACCAGAGTCCCAAGT-3' and reverse, 5'-CCCTAT GTGAAGCCCTATTT-3'; GLI3 forward, 5'-GAAGTGCTC CACTCGAACAGA-3' and reverse, 5'-GTGGCTGCATAG TGATTGCG-3'; cluster of differentiation (CD)133 forward, 5'-GAAAAGTTGCTCTGCGAACC-3' and reverse, 5'-CTC GACCTCTTTTGCAATCC-3'; SRY box-2 (SOX-2) forward, 5'-GGGAAATGGAGGGGTGCAAAAGAGG-3' and reverse, 5'-TTGCGTGAGTGTGGATGGGATTGGTG-3'; Nanog forward, 5'-TCC TCCTCT TCCTCTATACTA AC-3' and reverse, 5'-CCCACAATCACAGGCATAG-3'; $\beta$-actin forward 5'-AAGGGACTTCCTGTAACAATGCA-3' and reverse, 5'-CTGGAACGGTGAAGGTGACA-3'; miR-361-3p forward, 5'-ACACTCCAGCTGGGTCCCCCAGGTGTGATTC-3' and reverse, 5'-CTCAACTGGTGTCGTGGAGTCGGCAATTCA GTTGAGAAATCAGA-3'.

$R B$ cells and cell transfection. Two human RB cell lines (Y79 and Weri-Rb-1) were obtained from The American Type Culture Collection (Manassas, VA, USA) and cultured in RPMI-1640 medium (Gibco; Thermo Fisher Scientific, Inc.) supplemented with $10 \%$ fetal bovine serum (Gibco; Thermo Fisher Scientific, Inc.), penicillin $(100 \mathrm{U} / \mathrm{ml})$ and streptomycin $(100 \mathrm{U} / \mathrm{ml})$. Cells were then left to grow in a humidified incubator containing $5 \%$ $\mathrm{CO}_{2}$ at $37^{\circ} \mathrm{C}$. miR-361-3p, control miR or miR-361-3p inhibitor (all miRs, $100 \mu \mathrm{M}$; all miRs, Shanghai GenePharma Co., Ltd., Shanghai, China) were transfected using Lipofectamine 2000 (Invitrogen; Thermo Fisher Scientific, Inc.) according to the manufacturer's protocol. Following 48 h, subsequent experiments were performed. The sequences of miR-361-3p and control miR were as follows: miR-361-3p, 5'-UCCCCCAGG UGUGAUUCUGAUUU-3'; control miR, 5'-UUCUCCGAA CGUGUCACGUTT-3'. 
Table I. Clinical characteristics of patients with RB.

\begin{tabular}{clccccc}
\hline Case & Gender & Age (months) & Optic nerve invasion & Choroidal invasion & Extraocular invasion & Differentiation \\
\hline 1 & Male & 23 & Yes & Yes & No & Poor \\
2 & Male & 25 & No & No & No & Moderate \\
3 & Female & 13 & Yes & Yes & No & Moderate \\
4 & Male & 4 & Yes & Yes & No & Poor \\
5 & Male & 16 & Yes & Yes & No & Poor \\
6 & Male & 22 & Yes & Yes & No & Poor \\
7 & Female & 7 & Yes & No & No & Moderate \\
8 & Male & 19 & Yes & No & No & Poor \\
9 & Female & 8 & Yes & Yes & No & Moderate \\
10 & Female & 10 & Yes & Yes & No & Poor \\
\hline
\end{tabular}

MTT assay. An MTT assay was utilized to assess RB cell proliferation. Y79 and Weri-Rb-1 cells $\left(5 \times 10^{3}\right.$ cells per well) were seeded in 96-well plates. At 24, 48, 72 and $96 \mathrm{~h}$ following transfection, MTT (Beyotime Institute of Biotechnology, Haimen, China) at a final concentration of $0.5 \mathrm{mg} / \mathrm{ml}$ was added to each well and cultured at $37^{\circ} \mathrm{C}$ for $4 \mathrm{~h}$. The solution was removed and the precipitates were dissolved in dimethyl sulfoxide. Absorbance was measured at $570 \mathrm{~nm}$ using a microplate reader.

Cell counting kit-8 (CCK-8) assay. RB cell proliferation was assessed using A CCK-8 assay (Beyotime Institute of Biotechnology) was performed to confirm the results of proliferation, according to the manufacturer's protocol. Y79 and Weri-Rb-1 cells were seeded into 96 -well plates $\left(5 \times 10^{3}\right.$ cells per well). Following 48 and $96 \mathrm{~h}$ of transfection, the CCK-8 reagent was added to each well (dilution, 1:10) and cells were incubated for $2 \mathrm{~h}$ at $37^{\circ} \mathrm{C}$. Absorbance was measured at a $450 \mathrm{~nm}$ using a microplate reader.

Western blotting. Cells were harvested using radioimmunoprecipitation assay lysis buffer (Beyotime Institute of Biotechnology) and protein concentration was determined using the BCA method (Beyotime Institute of Biotechnology). Total protein $(20 \mu \mathrm{g})$ was separated using SDS-PAGE (10\% gels) and electrophoretically transferred onto nitrocellulose membranes (Bio-Rad Laboratories, Inc., Hercules, CA, USA). Subsequently, membranes were blocked with 5\% non-fat milk solution for $1 \mathrm{~h}$ at room temperature, and then incubated with the following primary and secondary antibodies (all Santa Cruz Biotechnology, Inc., Dallas, TX, USA) at $4^{\circ} \mathrm{C}$ overnight: GLI1 (cat. no. sc-20687; 1:500), GLI3 (cat. no. sc-20688; 1:500), CD133 (cat. no. sc-19365; 1:500), SOX-2 (cat. no. sc-365823; 1:500), Nanog (cat. no. sc-374103; $1: 500$ ) and $\beta$-actin (cat. no. sc-47778; 1:1,000). Subsequently, the membranes were incubated with horseradish peroxidase-conjugated immunoglobulin G secondary antibodies (horseradish peroxidase conjugated goat anti-rabbit Immunoglobulin G, cat. no. sc-2004 and horseradish peroxidase conjugated goat anti-mouse Immunoglobulin G, cat. no. sc-2005; each, 1:10,000; each, Santa Cruz Biotechnology, Inc.) for $1 \mathrm{~h}$ at room temperature. Membranes were then washed and proteins were visualized using an enhanced chemiluminescence kit (Beyotime Institute of Biotechnology) and exposed to X-ray film (Eastman Kodak, Rochester NY, USA). ImageJ software (version 1.48; National Institutes of Health, Bethesda, MD, USA) was used for densitometry.

Tumor sphere formation assay. Y79 and Weri-Rb-1 cells $\left(5 \times 10^{3}\right.$ cells per well) were seeded into ultra-low attachment 24-well plates (Corning Incorporated, Corning, NY, USA). All cells were maintained in serum-free RPMI-1640 medium (Gibco; Thermo Fisher Scientific, Inc.), containing $20 \mathrm{ng} / \mathrm{ml}$ epidermal growth factor (Invitrogen; Thermo Fisher Scientific, Inc.) and $10 \mathrm{ng} / \mathrm{ml}$ basic fibroblast growth factor (Invitrogen; Thermo Fisher Scientific, Inc.). Following 7 days of culture in a humidified incubator containing $5 \% \mathrm{CO}_{2}$ at $37^{\circ} \mathrm{C}$, tumor spheres were counted and images were captured at a magnification of $\mathrm{x} 200$ using a light microscope (Nikon Corporation, Tokyo, Japan).

TargetScan online tool analysis. The TargetScan online tool (http://www.targetscan.org/vert_72/) was utilized to predict the targets of the miRNAs being assessed.

Luciferase assay. The 3' untranslated (UTR) regions of GLI1 and GLI3 containing putative miR-361-3p binding sites were constructed based on the psiCHECK- 2 plasmid (Promega Corporation, Madison, WI, USA). Each plasmid was co-transfected with miR-361-3p or control miR (miR without targets) in RB cells using Lipofectamine 2000 (Invitrogen; Thermo Fisher Scientific, Inc.). Luciferase and Renilla signals were measured $48 \mathrm{~h}$ following co-transfection using a Dual-Luciferase Reporter Assay kit (Promega Corporation) according to the manufacturer's protocol and normalized against the activity of Renilla luciferase activity.

Statistical analysis. Each experiment was performed in triplicate and repeated at least three times. Data are expressed as the mean \pm standard deviation and were analyzed using a Student's t-test or one-way analysis of variance followed by a Tukey's multiple comparison test. A Pearson's correlation analysis performed to assess the correlation between miR-361-3p and Gli1 expression. Statistically significant differences were 
A

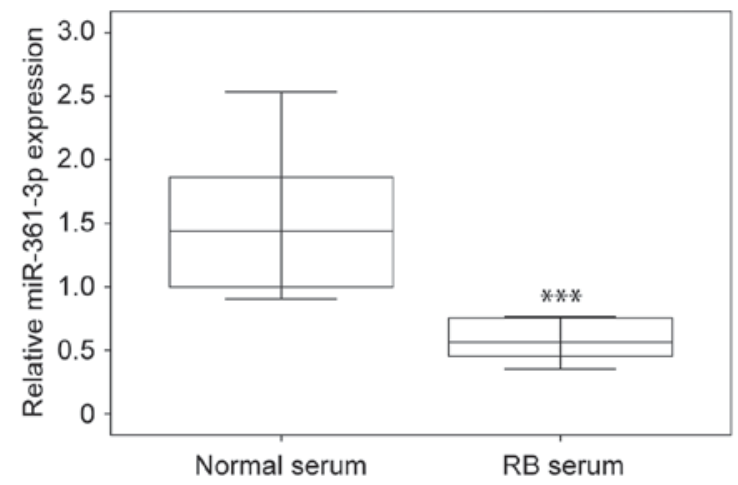

B

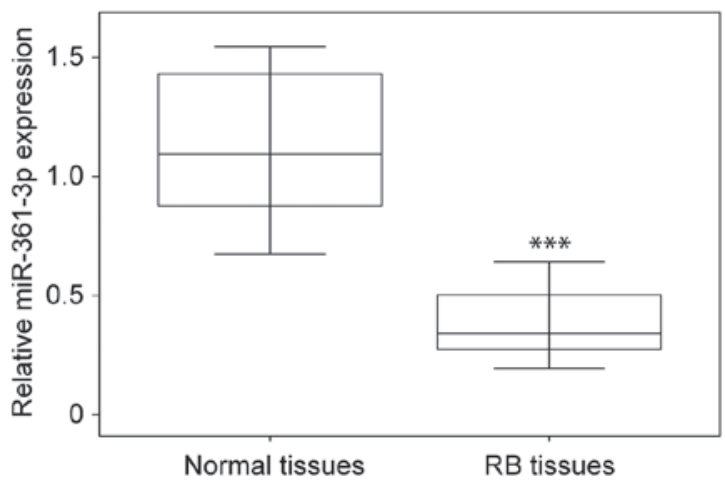

Figure 1. Expression of miR-361-3p is downregulated in the serum and tissue of patients with RB. (A) Reverse transcription-quantitative polymerase chain reaction analysis of miR-361-3p expression in the serum of 10 patients with $R B$ and 10 healthy participants. (B) miR-361-3p expression in $R B$ samples and normal tissue obtained from patients with RB. ${ }^{* * *} \mathrm{P}<0.001$ vs. normal serum or normal tissues. miR, microRNA; RB, retinoblastoma.

determined using SPSS version 16 software (SPSS, Inc., Chicago, IL, USA). P $<0.05$ was considered to indicate a statistically significant result.

\section{Results}

Expression of miR-361-3p is downregulated in the serum and tumor tissues of patients with $R B$. serum and tumor samples were obtained from 10 patients with RB and 10 healthy participants to assess the expression of miR-361-3p. The results revealed that the expression of miR-361-3p was significantly downregulated in RB serum (Fig. 1A). Furthermore, compared with normal adjacent tissues, miR-361-3p levels were significantly decreased in RB samples (Fig. 1B). These results indicate that miR-361-3p levels are decreased miR-361-3p RB patient serum and tumor samples.

miR-361-3p inhibits $R B$ cell proliferation. To study the function of miR-361-3p in RB, functional assays were performed in Y79 and Weri-Rb-1 cell lines. The results demonstrated that the expression of miR-361-3p was increased in RB cell lines transfected with miR-361-3p when compared with control miR (Fig. 2A). Cell malignancies between the miR-361-3p and control miR groups were also compared. An MTT cell proliferation assay was performed following Y79 and Weri-Rb-1 cell transfection with miR-361-3p or control miR. The results revealed that miR-361-3p significantly reduced $\mathrm{RB}$ cell proliferation (Fig. 2B). Cell proliferation was further analyzed using a CCK-8 assay. The results revealed that miR-361-3p inhibited Y79 and Weri-Rb-1 cell proliferation (Fig. 2C). Furthermore, miR-361-3p was inhibited using a miR-361-3p inhibitor (Fig. 2D). The results of the proliferation assays revealed that the inhibition of miR-361-3p significantly promoted RB cell proliferation (Fig. 2E and F). These results indicate that miR-361-3p reduces $\mathrm{RB}$ cell proliferation.

miR-361-3p restrains $R B$ cell stemness. In order to investigate the effect of miR-361-3p on RB cell stemness, the expression of stem cell markers including CD133, SOX-2 and Nanog were assessed using RT-qPCR. The expression levels of the aforementioned genes were significantly decreased in miR-361-3p overexpressed RB cells (Fig. 3A). Western blotting further confirmed the reduction of stem cell markers in miR-361-3p-overexpressed RB cells (Fig. 3B). A tumor sphere assay was subsequently performed to detect the ability of RB stem cells to self-renew. The results revealed that miR-361-3p markedly reduced RB cell self-renewal (Fig. 3C). In addition, a miR-361-3p inhibitor was transfected into RB cells and cells markers were identified using RT-qPCR and western blotting. The results demonstrated that the inhibition of miR-361-3p significantly increased the expression of these markers (Fig. 4A and B). A tumor sphere assay was then performed and it was determined that the miR-361-3p inhibitor promoted RB cell self-renewal (Fig. 4C). The results indicated that miR-361-3p inhibited Y79 and Weri-Rb-1 cell stemness.

miR-361-3p directly targets GLI1 and GLI3. To assess the molecular mechanism underlying the effect of miR-361-3p on RB cells, potential miR-361-3p targets were predicted using TargetScan online tool which identified two conserved binding sites of miR-361-3p in the 3'UTR of GLI1 and GLI3 mRNA (Fig. 5A). The current study hypothesized that GLI1 and GLI3 were potential targets of miR-361-3p due to their function in $\mathrm{RB}$ development. To confirm this, a luciferase reporter assay was performed using a psiCheck-2 reporter with wild-type of GLI1 or GLI3 3'UTR. The results revealed that miR-361-3p significantly inhibited luciferase activity (Fig. 5B). The protein expression levels of GLI1 and GLI3 were assessed in Y79 and Weri-Rb-1 cells following miR-361-3p transfection. As presented in Fig. 4C, GLI1 and GLI3 expression levels in Y79 and Weri-Rb-1 cells were reduced following miR-361-3p transfection. Together, these results demonstrated that GLI1 and GLI3 are miR-361-3p targets.

Negative correlation between miR-361-3p and GLII/3 in RB samples. A Pearson's correlation analysis was performed to assess miR-361-3p and its targets, GLI1 and GLI3. As presented in Fig. 6, a negative correlation was observed between miR-361-3p and GLI1/3 in RB tissues.

\section{Discussion}

According to a previous study, miR-361 has been implicated in the development and progression of various types of 
A
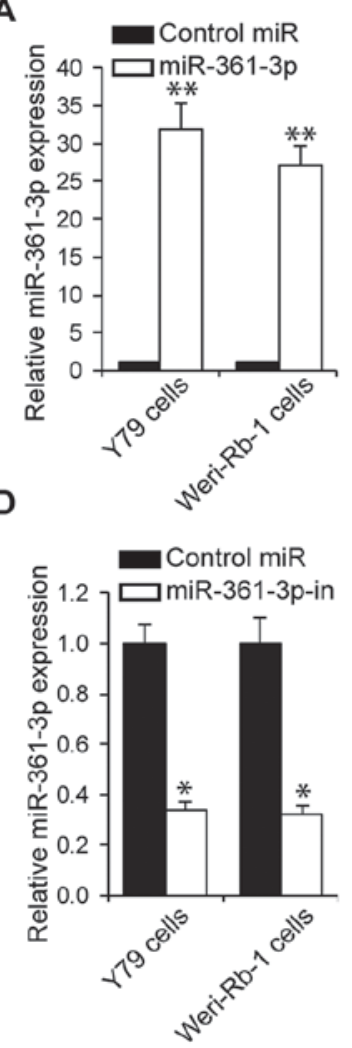

B

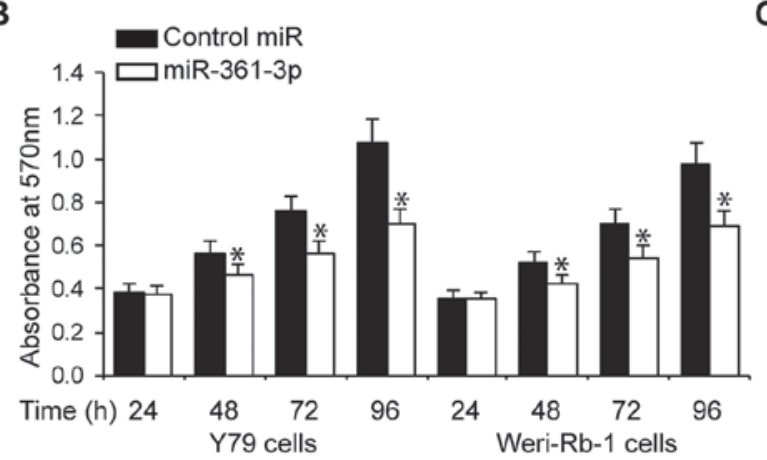

E

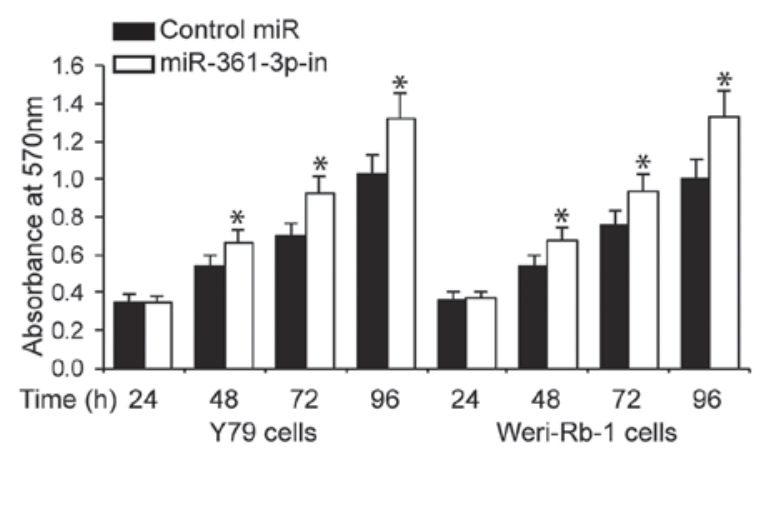

C

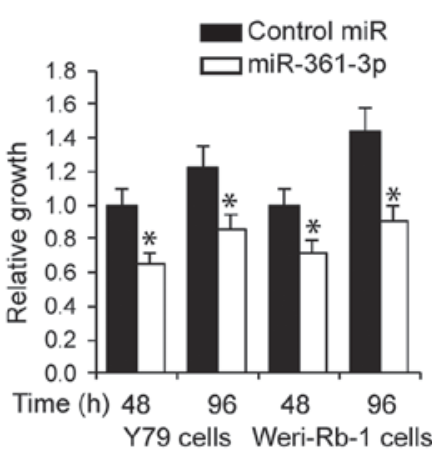

$\mathbf{F}$

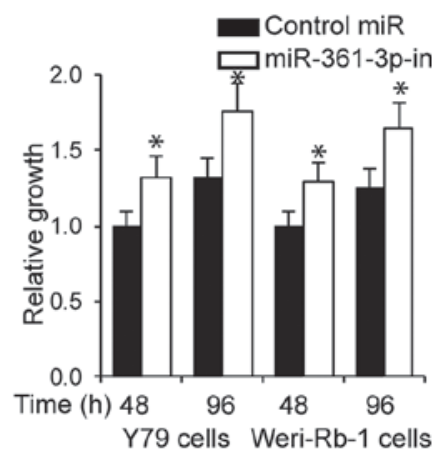

Figure 2. miR-361-3p inhibits RB cell proliferation. (A) RT-qPCR analysis of miR-361-3p expression in Y79 and Weri-Rb-1 RB cells following transfection with miR-361-3p or control miR for $48 \mathrm{~h}$. (B) An MTT assay was performed in Y79 and Weri-Rb-1 RB cells that exhibited miR-361-3p overexpression. (C) Relative cell proliferation was determined using a CCK-8 assay. (D) RT-qPCR analysis of miR-361-3p inhibitor expression in Y79 and Weri-Rb-1 RB cells following transfection with a miR-361-3p inhibitor or control miR for $48 \mathrm{~h}$. (E) An MTT assay was performed following miR-361-3p inhibition. (F) Relative cell growth was assessed using a CCK-8 assay following miR-361-3p inhibition. " $\mathrm{P}<0.05$ and ${ }^{* *} \mathrm{P}<0.01$ vs. control miR. miR, microRNA; RB, retinoblastoma; RT-qPCR, reverse transcription-quantitative polymerase chain reaction; CCK-8, cell counting kit-8; in, inhibitor.

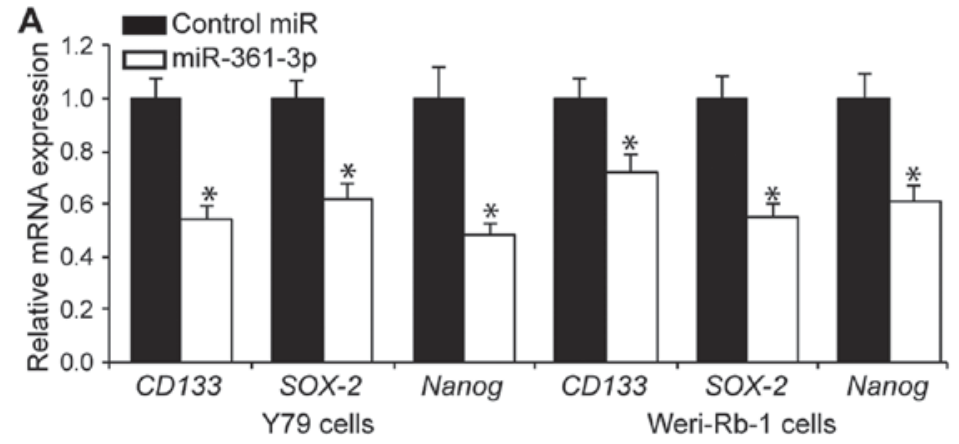

C

Y79 cells

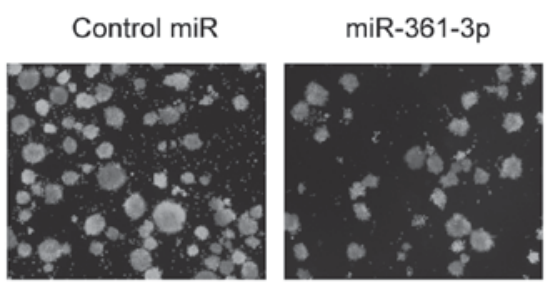

B

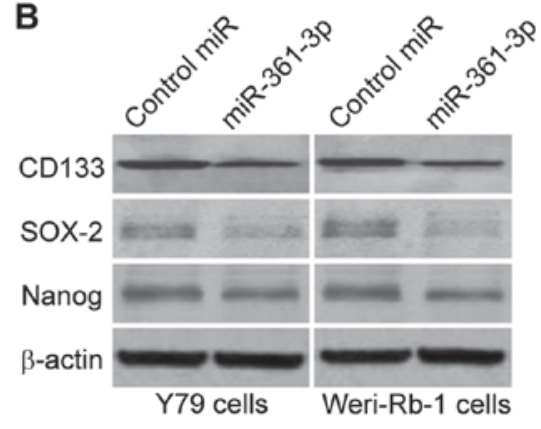

Weri-Rb-1 cells

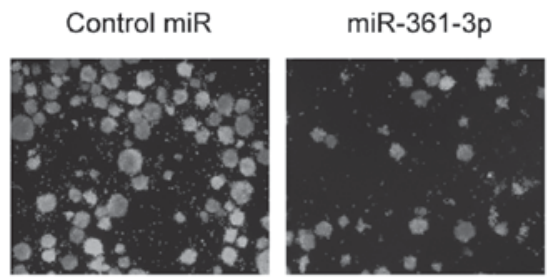

Figure 3. miR-361-3p overexpression reduces RB cell stemness. (A) Reverse transcription-quantitative polymerase chain reaction and (B) western blotting of the expression level of stem cell markers (CD133, SOX-2 and Nanog) in Y79 and Weri-Rb-1 RB cells transfected with miR-361-3p. (C) A tumor sphere assay was performed in Y79 and Weri-Rb-1 RB cells transfected with miR-361-3p (magnification, $\mathrm{x} 200$ ). " $\mathrm{P}<0.05$ vs. control miR. miR, microRNA; RB, retinoblastoma; $\mathrm{CD}$, cluster of differentiation; SOX-2, SRY box-2. 


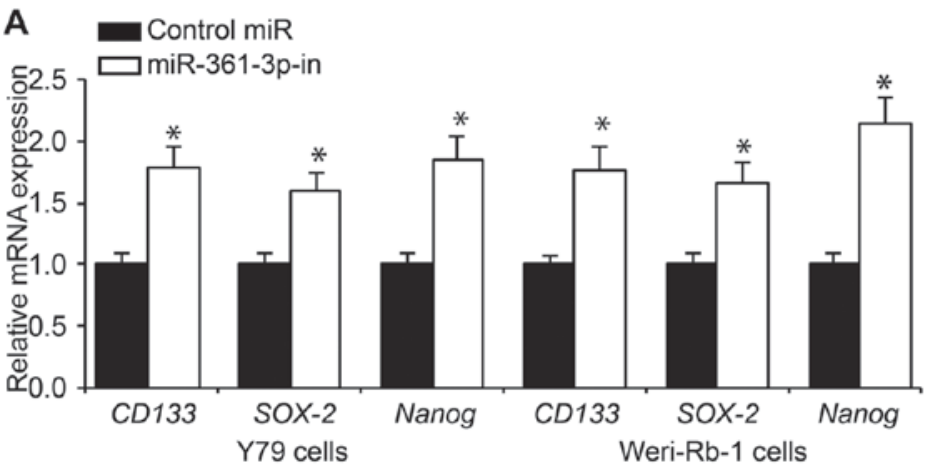

C

Y79 cells

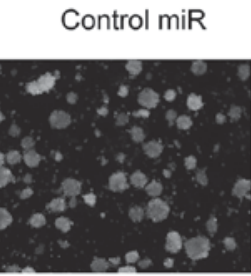

miR-361-3p-in

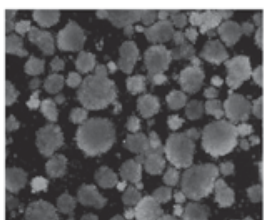

B
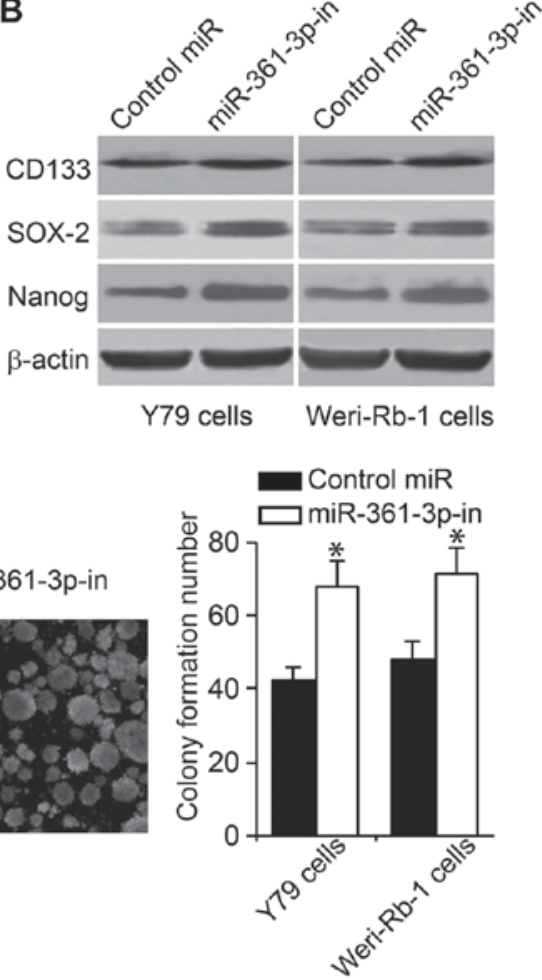

Figure 4. miR-361-3p inhibition promotes RB cell stemness. (A) Reverse transcription-quantitative polymerase chain reaction analysis and (B) western blotting of stem cell marker (CD133, SOX-2 and Nanog) expression in Y79 and Weri-Rb-1 RB cells transfected with a miR-361-3p inhibitor. (C) A tumor sphere assay was performed in Y79 and Weri-Rb-1 RB cells transfected with a miR-361-3p inhibitor (magnification, x200). "P<0.05 vs. control miR. miR, microRNA; RB, retinoblastoma; CD, cluster of differentiation; SOX-2, SRY box-2; in, inhibition.

A

\begin{tabular}{|c|c|}
\hline & $\begin{array}{l}\text { Predicted consequential pairing of target region (top) and } \\
\text { miRNA (bottom) }\end{array}$ \\
\hline & 5' ... UOCLUOCAGAAAAN---UCGGGGAG... \\
\hline $\begin{array}{l}\text { hsa-miR-361-3p } \\
\text { Position } 118-124 \text { of GL1 } 33^{\prime} \text { UTR }\end{array}$ & $\begin{array}{l}\text { UUUAGUCUUAGUGUCGACOCOCU } \\
\text {...GAUGGGGGUAUGGGCUCGGGC }\end{array}$ \\
\hline & UUUAGUCUUAGUGUGGACCCCCU \\
\hline
\end{tabular}

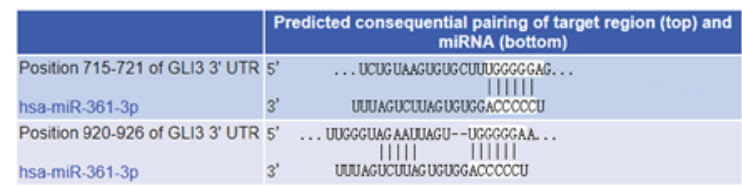

B
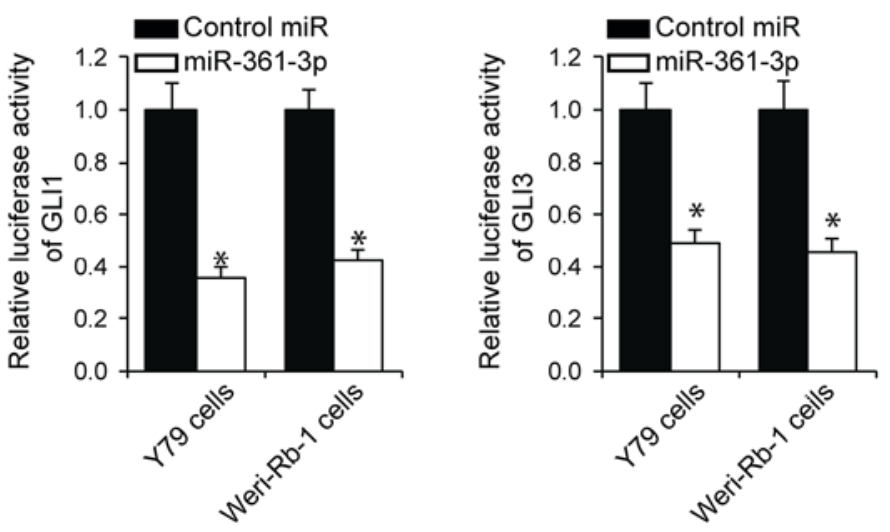

C

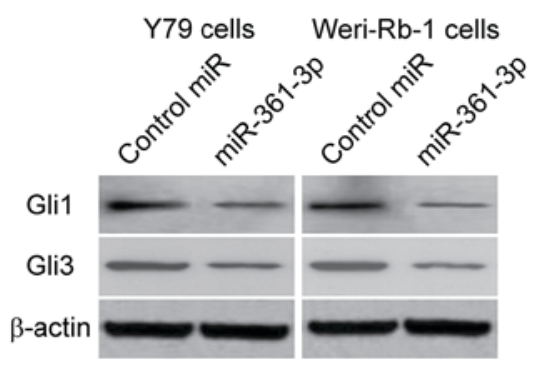

Figure 5. miR-361-3p directly targets GLI1 and GLI3. (A) A graphical representation of miR-361-3p binding sites in wild-type GLI1 and GLI3 3'-UTRs. (B) A luciferase assay was performed in Y79 and Weri-Rb-1 RB cells transfected with miR-361-3p and GLI1 or GLI3 3'UTR luciferase reporter plasmids. (C) Western blotting was performed to assess GLI1 and GLI3 expression in Y79 and Weri-Rb-1 RB cells transfected with miR-361-3p. "P<0.05 vs. control miR. miR, microRNA; RB, retinoblastoma; GLI, glioma-associated oncogene homologue; UTR, untranslated region.

cancer (21). In the present study, it was determined that the expression of miR-361-3p was 0.5 -fold lower in the serum of patients with RB compared with normal serum and 0.33 -fold lower in RB tumor tissues in comparison with healthy control samples. These results are consistent with a previous study by Chen et al (21), who also reported a decreased miR-361 expression profile in NSCLC tissues compared with normal lung tissue, and an inverse association with advanced stage and 

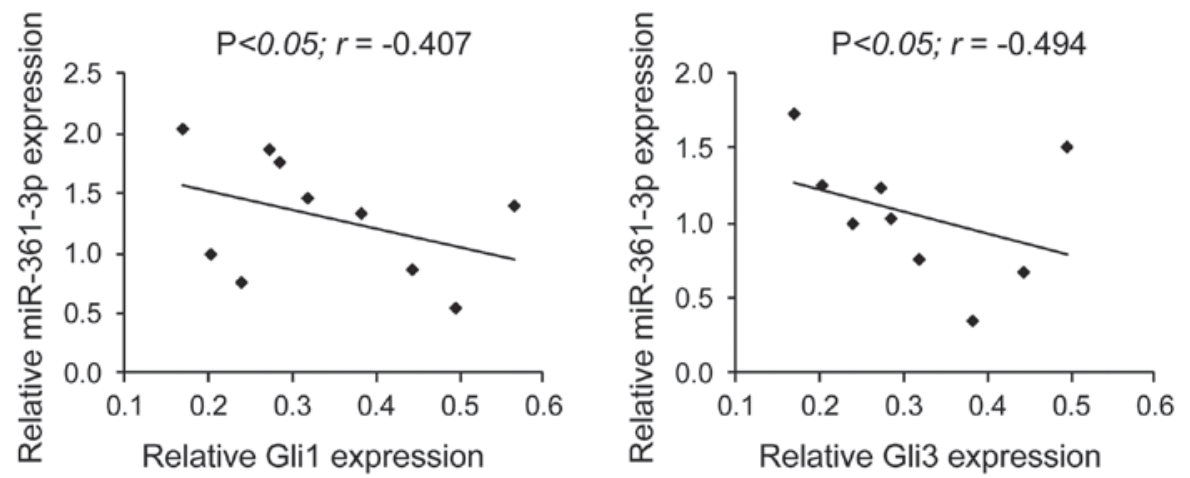

Figure 6. Negative correlation between miR-361-3p and GLI1/3 in RB samples. A Pearson's correlation analysis was performed between miR-361-3p and GLI1/3 in 10 RB tissues GLI1, P<0.05, $r=-0.407$; GLI1, P<0.05, $r=-0.494$. miR, microRNA; GLI, glioma-associated oncogene homologue; RB, retinoblastoma.

lymph node metastasis. Furthermore, it has been demonstrated that miR-361 is downregulated in colorectal carcinoma, gastric cancer (18) and castration-resistant prostate cancer (17), indicating that miR-361-5p may serve a tumor-suppressive miR role. However, miR-361-5p has been revealed to serve as an oncogene in cervical cancer progression by enhancing cell proliferation and invasion (16).

In the current study, Y79 and Weri-Rb-1 cells were utilized. However, these cell lines are in suspension and are thus difficult to use for the assessment of cell migration and invasion $(6,28)$. For this reason, the current study only assessed cell proliferation and stemness, which serves as a limitation. Despite this, the results demonstrated that miR-361-3p inhibits RB cell proliferation in two human RB cell lines (Y79 and Weri-Rb-1). Similarly, the CCK- 8 assay results revealed that miR-361-3p inhibited the proliferation of Y79 and Weri-Rb-1 cells. Montoya et al (4) also investigated the miR-mediated regulation of $\mathrm{RB}$ proliferation. It was demonstrated that miR-31 and/or miR-200a overexpression restricted the expansion of a highly proliferative cell line (Y79), but did not affect the growth rate of a less aggressive cell line (Weri-Rb-1) (4).

Cancer cell stemness is a key feature of cancer progression and often determines patient survival $(29,30)$. Common stemness characteristics of stem and cancer stem cells (CSCs) include self-renewal, differentiation potential and stemness involves the organization of a microenvironment that protects stem cell niche or stem cells $(31,32)$. Specifically, CSCs are a group of cells within the tumor mass, which exhibit stemness properties that promote cancer progression, including involvement in self-renewal cloning, growing, metastasizing, homing and re-proliferating (30). In addition, CSCs may influence the nutrient supply delivery of neighboring cells and cooperate in immune system elusion (33). They may also increase heterogeneous cell populations, which exhibit a high plasticity potential (33) and a high resistance to unfavorable factors within the tumor microenvironment, and may induce cell death (34) and quiescence (21).

miRs regulate diverse basic cellular functions, including proliferation, migration and invasion (35). Therefore, in the current study, RB cell lines were transfected with miR-361-3p, following which cell stemness ability was assessed via the detection of stem cell marker expression. The results demonstrated that miR-361-3p overexpression downregulated the expression of CD133, SOX-2 and Nanog. In addition, miR-361-3p significantly decreased RB cell self-renewal. Therefore, it was concluded that miR-361-3p inhibits the stemness ability of Y79 and Weri-Rb-1 cells. The key pathogenic mechanism in the development of $\mathrm{RB}$ is the inactivation of two RB gene alleles; a regulator of the G1 and S phase of the cell-cycle (36). Despite previous studies, there is currently no clinically acceptable biomarker that identifies the risk of RB metastasis (25). Different signaling pathways may be of great relevance to the oncogenesis and development of RB. Li et al (12) demonstrated that miR-497 overexpression decreases the proliferation, migration and invasion of human RB cells by targeting vascular endothelial growth factor A. Furthermore, Bai et al (11) revealed that miR-125b promotes tumor growth and suppresses apoptosis by targeting DNA Damage Regulated Autophagy Modulator 2 in RB. SHH signaling is another major pathway that is dysregulated in $\mathrm{RB}(6,25)$. SHH, a vertebrate homologue of the Drosophila segment polarity gene product, hedgehog, is associated with retina cell proliferation and differentiation during the process of embryonic development $(37,38)$. SHH-associated pathways may proceed directly or indirectly by activating a network of cascades $(39,40)$, which affect the apoptosis and metastasis of tumor cells (41) in various types of malignancy, including in pancreatic cancer (42) or ovarian cancer (43). Additionally, Song et al (6) demonstrated that the blockade of SHH signaling decreases RB cell viability and induces apoptosis by activating the phosphoinositide-3 kinase (PI3K)/protein kinase B (Akt) pathway. Additionally, previous studies have implied that the potential effect of the PI3K/Akt pathway on SHH signaling is regulated by GLI-dependent transcriptional activity (44). This is concordant with the current study as miR-361-3p was revealed to significantly reduce the expression of GLI1 and GLI3 protein in Y79 and Weri-Rb-1 cells. Thus, the results demonstrated that GLI1 and GLI3 are targets of miR-361-3p and that a negative correlation exists between miR-361-3p and GLI1/3 in RB samples. However, the absence of a non-cancerous cell line as a control is a limitation of the present study. Further assessment is therefore required to confirm these results.

In conclusion, the results of the present study indicate that $\mathrm{miR}-361-3 \mathrm{p}$ regulates the proliferation and metastasis of RB cells by targeting SHH signaling. The current study also revealed that $\mathrm{SHH}$ signaling serves an important role in the development of RB and that miR-361-3p should be assessed as a potential therapeutic target for RB treatment. The results of the present study may also enhance the development of 
targeted therapeutic approaches for RB and improve the efficacy of current RB management. However, further preclinical studies are necessary to determine the role of the miR-361-3p in different malignant tumors and to reveal $\mathrm{SHH}$-associated signaling transduction.

\section{Acknowledgements}

Not applicable.

\section{Funding}

No funding was received.

\section{Availability of data and materials}

All data and materials supported the results of the present study are available in the published article.

\section{Authors' contributions}

DZ and ZC performed experiments, and collected and analyzed the data. DZ wrote the manuscript and ZC designed the experiments. Each author read the manuscript and approved the final version of the manuscript.

\section{Ethics approval and consent to participate}

This study was approved by the Clinical Research Ethics Committee of The Third Affiliated Hospital of Qiqihar Medical University.

\section{Patient consent for publication}

Written informed consent obtained from all patients prior to publication.

\section{Competing interests}

The authors declare that they have no conflicts of interests.

\section{References}

1. Mahoney MC, Burnett WS, Majerovics A and Tanenbaum H: The epidemiology of ophthalmic malignancies in New York State. Ophthalmology 97: 1143-1147, 1990.

2. Shields CL and Shields JA: Diagnosis and management of retinoblastoma. Cancer Control 11: 317-327, 2004.

3. Fabian ID, Onadim Z, Karaa E, Duncan C, Chowdhury T, Scheimberg I, Ohnuma SI, Reddy MA and Sagoo MS: The management of retinoblastoma. Oncogene 37: 1551-1560, 2018.

4. Montoya V, Fan H, Bryar PJ, Weinstein JL, Mets MB, Feng G Martin J, Martin A, Jiang H and Laurie NA: Novel miRNA-31 and miRNA-200a-mediated regulation of retinoblastoma proliferation. PLoS One 10: e0138366, 2015.

5. Liu Q, Wang Y, Wang H, Liu Y, Liu T and Kunda PE: Tandem therapy for retinoblastoma: Immunotherapy and chemotherapy enhance cytotoxicity on retinoblastoma by increasing apoptosis. J Cancer Res Clin Oncol 139: 1357-1372, 2013.

6. Song Z, Du Y and Tao Y: Blockade of sonic hedgehog signaling decreases viability and induces apoptosis in retinoblastoma cells: The key role of the PI3K/Akt pathway. Oncol Lett 14: 4099-4105, 2017.

7. Lytle JR, Yario TA and Steitz JA: Target mRNAs are repressed as efficiently by microRNA-binding sites in the 5'UTR as in the 3'UTR. Proc Natl Acad Sci USA 104: 9667-9672, 2007.
8. Zheng H, Zhang F, Lin X, Huang C, Zhang Y, Li Y, Lin J, Chen W and Lin X: MicroRNA-1225-5p inhibits proliferation and metastasis of gastric carcinoma through repressing insulin receptor substrate- 1 and activation of $\beta$-catenin signaling. Oncotarget 7 : 4647-4663, 2016

9. Sestini S, Boeri M, Marchiano A, Pelosi G, Galeone C, Verri C, Suatoni P, Sverzellati N, La Vecchia C, Sozzi G and Pastorino U: Circulating microRNA signature as liquid-biopsy to monitor lung cancer in low-dose computed tomography screening. Oncotarget 6: 32868-32877, 2015.

10. Yang $\mathrm{Y}$ and Mei Q: miRNA signature identification of retinoblastoma and the correlations between differentially expressed miRNAs during retinoblastoma progression. Mol Vis 21: 1307-1317, 2015.

11. Bai S, Tian B, Li A, Yao Q, Zhang G and Li F: MicroRNA-125b promotes tumor growth and suppresses apoptosis by targeting DRAM2 in retinoblastoma. Eye (Lond) 30: 1630-1638, 2016.

12. Li J, Zhang Y, Wang X and Zhao R: microRNA-497 overexpression decreases proliferation, migration and invasion of human retinoblastoma cells via targeting vascular endothelial growth factor A. Oncol Lett 13: 5021-5027, 2017.

13. He B, Xu Z, Chen J, Zheng D, Li A and Zhang LS: Upregulated microRNA-143 inhibits cell proliferation in human nasopharyngeal carcinoma. Oncol Lett 12: 5023-5028, 2016.

14. Castro-Magdonel BE, Orjuela M, Camacho J, García-Chéquer AJ, Cabrera-Muñoz L, Sadowinski-Pine S, Durán-Figueroa N, Orozco-Romero MJ, Velázquez-Wong AC, Hernández-Ángeles A, et al: miRNome landscape analysis reveals a 30 miRNA core in retinoblastoma. BMC Cancer 17: 458, 2017.

15. Kanitz A, Imig J, Dziunycz PJ, Primorac A, Galgano A, Hofbauer GF, Gerber AP and Detmar M: The expression levels of microRNA-361-5p and its target VEGFA are inversely correlated in human cutaneous squamous cell carcinoma. PLoS One 7: e49568, 2012.

16. Wu X, Xi X, Yan Q, Zhang Z, Cai B, Lu W and Wan X: MicroRNA-361-5p facilitates cervical cancer progression through mediation of epithelial-to-mesenchymal transition. Med Oncol 30: 751, 2013.

17. Liu D, Tao T, Xu B, Chen S, Liu C, Zhang L, Lu K, Huang Y, Jiang L, Zhang X, et al: MiR-361-5p acts as a tumor suppressor in prostate cancer by targeting signal transducer and activator of transcription-6(STAT6). Biochem Biophys Res Commun 445: 151-156, 2014

18. Ma F, Song H, Guo B, Zhang Y, Zheng Y, Lin C, Wu Y, Guan G, Sha R, Zhou Q, et al: MiR-361-5p inhibits colorectal and gastric cancer growth and metastasis by targeting staphylococcal nuclease domain containing-1. Oncotarget 6: 17404-17416, 2015.

19. Sun JJ, Chen GY and Xie ZT: MicroRNA-361-5p inhibits cancer cell growth by targeting CXCR6 in hepatocellular carcinoma. Cell Physiol Biochem 38: 777-785, 2016.

20. Cao ZG, Huang YN, Yao L, Liu YR, Hu X, Hou YF and Shao ZM: Positive expression of miR-361-5p indicates better prognosis for breast cancer patients. J Thorac Dis 8: 1772-1779, 2016.

21. Chen W, Wang J, Liu S, Wang S, Cheng Y, Zhou W, Duan C and Zhang C: MicroRNA-361-3p suppresses tumor cell proliferation and metastasis by directly targeting SH2B1 in NSCLC. J Exp Clin Cancer Res 35: 76, 2016.

22. Kim JE, Singh RR, Cho-Vega JH, Drakos E, Davuluri Y, Khokhar FA, Fayad L, Medeiros LJ and Vega F: Sonic hedgehog signaling proteins and ATP-binding cassette G2 are aberrantly expressed in diffuse large B-cell lymphoma. Mod Pathol 22: 1312-1320, 2009.

23. Kim JE, Kim H, Choe JY, Sun P, Jheon S and Chung JH: High expression of Sonic hedgehog signaling proteins is related to the favorable outcome, EGFR mutation, and lepidic predominant subtype in primary lung adenocarcinoma. Ann Surg Oncol 20 (Suppl 3): S570-S576, 2013.

24. Taipale J and Beachy PA: The Hedgehog and Wnt signalling pathways in cancer. Nature 411: 349-354, 2001

25. Choe JY, Yun JY, Jeon YK, Kim SH, Choung HK, Oh S, Park M and Kim JE: Sonic hedgehog signalling proteins are frequently expressed in retinoblastoma and are associated with aggressive clinicopathological features. J Clin Pathol 68: 6-11, 2015.

26. Pang X, Shimizu A, Kurita S, Zankov DP, Takeuchi K, Yasuda-Yamahara M, Kume S, Ishida T and Ogita H: Novel Therapeutic role for dipeptidyl peptidase III in the treatment of hypertension. Hypertension 68: 630-641, 2016.

27. Livak KJ and Schmittgen TD: Analysis of relative gene expression data using real-time quantitative PCR and the 2(-Delta Delta C(T)) method. Methods 25: 402-408, 2001. 
28. Zhu X, Xue L, Yao Y, Wang K, Tan C, Zhuang M, Zhou F and Zhu L: The FoxM1-ABCC4 axis mediates carboplatin resistance in human retinoblastoma Y-79 cells. Acta Biochim Biophys Sin (Shanghai) 50: 914-920, 2018.

29. Colak S and Medema JP: Cancer stem cells-important players in tumor therapy resistance. FEBS J 281: 4779-4791, 2014.

30. Aponte PM and Caicedo A: Stemness in cancer: Stem cells, cancer stem cells, and their microenvironment. Stem Cells Int 2017: 5619472, 2017.

31. Visvader JE: Cells of origin in cancer. Nature 469: 314-322, 2011.

32. Visvader JE and Clevers H: Tissue-specific designs of stem cell hierarchies. Nat Cell Biol 18: 349-355, 2016.

33. Cabrera MC, Hollingsworth RE and Hurt EM: Cancer stem cell plasticity and tumor hierarchy. World J Stem Cells 7: 27-36, 2015.

34. Plaks V, Kong N and Werb Z: The cancer stem cell niche: How essential is the niche in regulating stemness of tumor cells? Cell Stem Cell 16: 225-238, 2015.

35. Wang L, Song G, Tan W, Qi M, Zhang L, Chan J, Yu J, Han J and Han B: MiR-573 inhibits prostate cancer metastasis by regulating epithelial-mesenchymal transition. Oncotarget 6: 35978-35990, 2015.

36. Godbout R, Dryja TP, Squire J, Gallie BL and Phillips RA: Somatic inactivation of genes on chromosome 13 is a common event in retinoblastoma. Nature 304: 451-453, 1983.

37. Wechsler-Reya R and Scott MP: The developmental biology of brain tumors. Annu Rev Neurosci 24: 385-428, 2001.

38. Milla LA, Gonzalez-Ramirez CN and Palma V: Sonic Hedgehog in cancer stem cells: A novel link with autophagy. Biol Res 45: 223-230, 2012
39. Javelaud D, Pierrat MJ and Mauviel A: Crosstalk between TGF- $\beta$ and hedgehog signaling in cancer. FEBS Lett 586: 2016-2025, 2012.

40. Yanai K, Nakamura M, Akiyoshi T, Nagai S, Wada J, Koga K, Noshiro H, Nagai E, Tsuneyoshi M, Tanaka M and Katano M: Crosstalk of hedgehog and Wnt pathways in gastric cancer. Cancer Lett 263: 145-156, 2008.

41. Kasperczyk H, Baumann B, Debatin KM and Fulda S: Characterization of sonic hedgehog as a novel NF-kappaB target gene that promotes NF-kappaB-mediated apoptosis resistance and tumor growth in vivo. FASEB J 23: 21-33, 2009.

42. Feldmann G, Dhara S, Fendrich V, Bedja D, Beaty R, Mullendore M, Karikari C, Alvarez H, Iacobuzio-Donahue C, Jimeno A, et al: Blockade of hedgehog signaling inhibits pancreatic cancer invasion and metastases: A new paradigm for combination therapy in solid cancers. Cancer Res 67: 2187-2196, 2007.

43. Ke Z, Caiping S, Qing Z and Xiaojing W: Sonic hedgehog-Gli1 signals promote epithelial-mesenchymal transition in ovarian cancer by mediating PI3K/AKT pathway. Med Oncol 32: 368, 2015.

44. Yoo YA, Kang MH, Lee HJ, Kim BH, Park JK, Kim HK, Kim JS and Oh SC: Sonic hedgehog pathway promotes metastasis and lymphangiogenesis via activation of Akt, EMT, and MMP-9 pathway in gastric cancer. Cancer Res 71: 7061-7070, 2011.

This work is licensed under a Creative Commons Attribution-NonCommercial-NoDerivatives 4.0 International (CC BY-NC-ND 4.0) License. 